Para citar este artículo: Perelló Oliver, S., \& Muela Molina, C. (2019). ¿De quién es esa voz? Evolución de su presencia y uso por tipo de personaje en la publicidad radiofónica (2009-2016). Anuario Electrónico de Estudios en Comunicación Social "Disertaciones", 12(2), 79-96. Doi: http://dx.doi.org/10.12804/revistas. urosario.edu.co/disertaciones/a.6786

\title{
¿DE QUIÉN ES ESA VOZ? EVOLUCIÓN DE SU PRESENCIA Y USO POR TIPO DE PERSONAJE EN LA PUBLICIDAD RADIOFÓNICA (2009-2016)*
}

\section{Whose Is that Voice? Evolution of its Presence and Use by Type of Spokesperson in Radio Advertising (2009-2016)}

De quem é essa voz? Evolução de sua presença e uso por tipo de personagem na publicidade radiofónica (2009-2016)

Salvador Perelló Oliver, Universidad Rey Juan Carlos (España)

salvador.perello@urjc.es

Clara Muela Molina, Universidad Rey Juan Carlos (España)

clara.muela@urjc.es

Recibido: 07 de mayo de 2018

Aprobado: 01 de julio de 2018

* Esta investigación ha sido desarrollada en el marco del proyecto Responsabilidad social, autorregulación publicitaria y salud en la radio, código (CSO2017-82267-R) financiado por el Ministerio de Economía, Industria y Competitividad, Secretaría de Estado de Investigación, Desarrollo e Innovación. 


\section{DISERTACIONES}

ESTUDIOS

El papel del sonido en la comunicación: contribución, funciones y efectos

ISSN: 1856-9536

Doi: http://dx.doi.org/10.12804/revistas.urosario.edu.co/disertaciones/a.6786

Volumen 12, Número 2 / Julio-diciembre 2019

Versión PDF para imprimir desde

http://revistas.urosario.edu.co/index.php/disertaciones

\section{RESUMEN}

El presente trabajo analiza el uso de la voz en la publicidad radiofónica a partir de tres objetivos concretos: cuantificar la presencia de los distintos tipos de voces; analizar la praxis de los anunciantes respecto a la elección de un tipo de voz concreto; y estudiar las formas de identificación del portavoz y su implicación con el producto a través del mensaje. El estudio se basa en dos muestras estadísticamente representativas referidas a los años 2009 y 2016. El análisis pone de manifiesto que son las voces de los consumidores -cuyo peso se ha incrementado en un $52 \%$ - y las propias de los empleados de las empresas - que se han multiplicado por 14- las que más presencia acumulan en las emisiones publicitarias radiofónicas entre los dos años de referencia. El trabajo constata la infrautilización de la radio como medio publicitario, avanzando como consecuencia, entre otros aspectos, el uso incorrecto e ineficaz de la voz para la identificación de determinados personajes que avalan al anunciante y se dirigen a la audiencia en su nombre.

Palabras clave: persuasión, publicidad, radio, voz.

\section{ABSTRACT}

This paper analyzes the use of voice in radio advertising based on three specific objectives: quantify the presence of different types of articulations, analyze the praxis of the advertisers regarding the choice of a particular voice type, and study the forms for identifying the spokesperson and their implication with the product through the message. The study presented is based on two statistically representative samples referring to the years 2009 and 2016. The analysis shows that the voices of consumers - whose weight has increased by $52 \%$ - and the employees of the companies - which have multiplied by 14 - are the most frequently broadcasted in radio advertising for the reference years. The work confirms that the radio is underused as an advertising medium, among other aspects, because it relates to different spokespeople who represent the advertiser and addresses the audience in their behalf.

Keywords: Persuasion, advertising, radio, voice.

\section{RESUMO}

O presente trabalho analisa o uso da voz na publicidade radiofónica a partir de três objetivos concretos: quantificar a presença dos distintos tipos de vozes; analisar a práxis dos anunciantes respeito à eleição de um tipo de voz concreto; e estudar as formas de identificação do porta-voz e sua implicação no produto através da mensagem. 0 estudo baseia-se em duas mostras estatisticamente representativas referidas aos anos 2009 e 2016. A análise põe de manifesto que são as vozes dos consumidores - cujo peso se tem incrementado em um $52 \%$ - e as próprias dos empregados das empresas - que se têm multiplicado por 14- as que mais presença acumulam nas emissões publicitárias entre os dois anos de referências. $O$ trabalho constata a subutilização da rádio como meio publicitário avançado como consequência entre outros aspetos, o uso incorreto e ineficaz da voz para a identificação de determinadas personagens que avaliam ao anunciante e dirigem-se à audiência em seu nome.

Palavras-chave: persuasão, publicidade, radio, voz. 


\section{DISERTACIONES}

ESTUDIOS

\section{Introducción}

Desde su origen, la publicidad radiofónica ha evolucionado muy poco. Es realista, racional, poco creativa y nada sugerente (Rodero Antón, 2008). Su forma de hacer es similar a la de décadas pasadas, con un discurso homogéneo donde categorías de productos tan dispares como las de finanzas o salud comparten estilos discursivos casi idénticos (Perelló Oliver \& Muela Molina, 2013). La dramatización, que es un exponente de la creatividad frente a estructuras narrativas informativas, solo está presente en un 1,2\% de las cuñas radiofónicas, mientras que en el $70 \%$ de los casos se construye sobre la base de una estructura narrativa informativa (Perona Páez, 2007), y el restante es un estilo mixto que combina la realidad con el drama. La imitación de contenidos informativos también es una constante, ya que casi 1 de cada 4 cuñas $(18,7 \%)$ imita algún género periodístico como la noticia, el reportaje, la entrevista, u otros programas radiofónicos como concursos o espacios de participación de la audiencia (Muela Molina, 2010). Y solo se recurre a la ficción en el $28,25 \%$ de los casos frente a un $71,75 \%$ que no la incluyen (Arcos Foix \& Perona Páez, 2011), a lo que cabe añadir que la gran mayoría de los mensajes publicitarios están protagonizados por un locutor anónimo que describe las características del producto detallando sus bondades (Muela Molina, 2001).

En esa forma tradicional y poco innovadora de hacer la publicidad radiofónica, la voz tiene un papel destacado, ya que aporta la información sobre el producto o servicio anunciado a través del texto, a la vez que identifica a los protagonistas del anuncio. En este sentido, son varios los mecanismos que se pueden utilizar para personificar la marca (Fleck, Michel \& Zeitoun, 2014). La clasificación más utilizada establece cuatro modalidades (Belch \& Belch, 2013; Fleck, et al., 2014; Friedman, Termini \& Washington, 1976; Khatri, 2006; Stafford, et al., 2002; Stephens \& Faranda, 1993): 1) famosos, 2) consumidor, 3) expertos profesionales y 4) portavoz de la empresa. La presencia de personajes en publicidad, en especial los famosos, ha recibido mucha atención por parte de los investigadores desde muchos y diferentes ámbitos, principalmente para demostrar que su inclusión en un mensaje publicitario incrementa su eficacia (Mittelstaedt, et al., 2000).

Estudios previos que han analizado la voz en la publicidad radiofónica se han centrado preferentemente en los efectos que esta provoca en el receptor según sus características prosódicas como el tono, el ritmo o el acento. También son numerosos los trabajos que han centrado su interés en el género de la voz y sus efectos comunicativos (Rodero, Larrea \& Vázquez, 2010), el uso de estereotipos vocales o sus representaciones sociales. Casos que se alejan mucho de nuestro objeto de estudio. Son menos los que han enfocado su análisis en la presencia de las voces según el género, que han permitido constatar el predominio de las masculinas sobre las femeninas (Muela Molina, 2001; Perona Páez \& Barbeito Veloso, 2008; Piñeiro Otero, 2011) o del tipo de voz según la función que ejerce en el mensaje o el tipo de prescriptor (Muela Molina, 2001; Piñeiro Otero, 2011). Sin embargo, no se ha encontrado ningún trabajo que analice la presencia de la voz según la persona a la que representa y por la forma de identificarse ante al oyente.

La exposición diaria a los medios masivos de comunicación muestra un claro incremento en el uso de personajes famosos en publicidad como una forma de añadir valor al producto y diferenciar marcas, especialmente en los 


\section{DISERTACIONES}

ESTUDIOS

El papel del sonido en la comunicación: contribución, funciones y efectos

ISSN: $1856-9536$

Doi: http://dx.doi.org/10.12804/revistas.urosario.edu.co/disertaciones/a.6786

Volumen 12, Número 2 / Julio-diciembre 2019

Versión PDF para imprimir desde

http://revistas.urosario.edu.co/index.php/disertaciones

medios gráficos, como las revistas o la televisión, donde la imagen y el atractivo del personaje juegan un papel destacado (Black \& Choi, 2013; Belch \& Belch, 2013; Choi, Lee \& Kim, 2005; Hsu \& McDonald, 2002; Stout \& Moon, 1990). Sin embargo, la presencia de los distintos personajes protagonistas de la publicidad identificados a través de sus voces no ha recibido mucha atención por parte de los investigadores en el medio radio. Por ello, el principal interés a la hora de desarrollar este estudio es el de proporcionar una valiosa aportación sobre el comportamiento del anunciante y sus preferencias a la hora de elegir una voz que encarne la marca y describa el producto, así como conocer la evolución de su uso en los últimos años en un medio publicitario importante, que no es la televisión o las revistas, que cuenta con la segunda mayor audiencia en España.

El punto de partida de este trabajo se asienta en los estudios precedentes que avalan la infrautilización de la radio como medio publicitario y aporta un análisis del uso incorrecto e ineficaz de la voz en cuanto representación de determinados personajes. Por lo tanto, el objetivo general es analizar la evolución del uso de la voz en la publicidad radiofónica. Además, los objetivos específicos se concretan en los siguientes: 1) cuantificar la presencia de los distintos tipos de voces según el personaje al que representan en los últimos años; 2) analizar la praxis de los anunciantes respecto a la elección de una voz; y 3) analizar la forma de identificación y la implicación del portavoz con el producto a través del mensaje.

\section{Marco y antecedentes teóricos y preguntas de investigación}

El Elaboration Likelihood Model (ELM) sugiere la existencia de una ruta central y otra periférica para la persuasión en publicidad (Petty \& Cacioppo, 1986). En la ruta central, el proceso cognitivo del mensaje es la base sobre la que el receptor piensa o analiza la información y los argumentos presentados en la publicidad. Así, la publicidad influye en la actitud del destinatario a través de su base de conocimiento, sus creencias y opiniones sobre el producto anunciado. Por otro lado, el uso de voces que remiten a determinadas personalidades puede actuar como una variable periférica debido a la actitud positiva o negativa hacia la persona o porque el destinatario del mensaje hace una simple inferencia sobre los méritos defendidos por la voz que representa al anunciante basada en simples factores (Petty, Cacioppo \& Schumann, 1983).

Otros trabajos se han basado en el modelo de la transferencia de significado (Amos, et al., 2008; Doss, 2011; Silvera \& Austad, 2004), que se fundamenta en que los personajes famosos añaden valor al producto por medio de este proceso en contraste con personajes o actores anónimos (McCracken, 1989). Por otra parte, los antecedentes que han analizado la presencia de famosos en publicidad han aplicado preferentemente el modelo de la credibilidad de la fuente (Ohanian, 1990) demostrando que la recomendación realizada por un experto o un no famoso es más efectiva que la realizada por una celebridad (Biswas, et al., 2006; Eisend \& Langner, 2010; Gaied \& Rached, 2010).

Sin embargo, en radio es frecuente el uso de una voz de la marca o straight announcer (Altstiel y Grow, 2006), que habla directamente al oyente como representante del anunciante. De esta forma, la audiencia siente como si se dirigiera a ella personalmente. El equivalente de este tipo de voz en televisión es un presentador desconocido o el llamado 'cabeza parlante'. En este caso, el efecto de este portavoz anónimo puede hacer que una marca se perciba como menos abstracta o anónima (Fleck, et al., 2012). 


\section{DISERTACIONES}

ESTUDIOS

El papel del sonido en la comunicación: contribución, funciones y efectos

ISSN: 1856-9536

Doi: http://dx.doi.org/10.12804/revistas.urosario.edu.co/disertaciones/a.6786

Volumen 12, Número 2 / Julio-diciembre 2019

Versión PDF para imprimir desde

http://revistas.urosario.edu.co/index.php/disertaciones

En otro sentido, determinados personajes con mayor reconocimiento social, como las celebridades, suponen una mayor inversión para un anunciante que los quiera contratar para su campaña, por lo que no todas las marcas pueden contar con un personaje famoso para su publicidad.

De este hecho, y siguiendo estudios previos (Belch \& Belch, 2013; Choi, et al., 2005; Stout \& Moon, 1990), deriva la necesidad de conocer la presencia de los distintos tipos de voces según categorías de producto con alta o baja implicación (Petty, Cacioppo \& Schumann, 1983) y los anunciantes que más los utilizan. Su estudio permitirá responder a los siguientes interrogantes que ha generado la investigación:

PI 1: ¿Cuál es la evolución en el uso de los diferentes tipos de voces según el tipo de anunciante?

PI 2: ¿Cuál es la evolución en el uso de los diferentes tipos de voces según el tipo de producto?

Cuando un oyente escucha una voz a través de la radio de la que no tiene referencia visual, recrea mentalmente las características físicas de la persona que habla y le atribuye al mismo tiempo determinadas cualidades de su personalidad (Knapp, 1980). La intención del anunciante al usar determinadas voces en publicidad es transmitir su confianza percibida y crear una imagen para la marca a través de la persona, en lugar de hacerlo a través del producto mismo (Kertz \& Ohanian, 1992). De otro lado, Priester y Petty (2003) sostienen que si el destinatario tiene cierta seguridad de que una fuente experta proporciona información precisa debido a su alta confiabilidad, puede renunciar a la tarea de analizar el mensaje, aceptando, así, de forma irreflexiva la conclusión como válida, hecho que constatan Buchholz y Smith (1991) al comprobar las reacciones de consumidores con alta implicación con determinados productos cuando son expuestos a la publicidad de radio. No obstante, en un medio donde no existe imagen física es necesario que el personaje que representa al anunciante se presente frente a su interlocutor y se identifique lo antes posible.

Por otra parte, es relevante tener en cuenta las formas lingüísticas, como los pronombres personales, que hacen referencia a las diversas figuras. El pronombre utilizado en la redacción del mensaje es otra variable que indica el grado de relación que establece el portavoz con el propio producto, marcando una mayor o menor distancia con él. Así, el pronombre de primera persona 'yo' se utiliza cuando el hablante se dirige directamente a su audiencia. El 'yo' también se denomina 'autor implícito' porque este presentador revela detalles personales sobre sí mismo. Lo que el 'yo' dice se supone que es 'verdadero' porque es una revelación sincera de un presentador que no tiene ninguna razón para mentir (Tulloch, 2014). La presencia de la primera persona del singular en un anuncio de radio se relaciona con la idiosincrasia de un medio donde un narrador, a menudo, habla confidencialmente a un oyente de una manera íntima (Stern, 1991). También se recurre a la tercera persona del singular mediante los pronombres personales tónicos 'él' o 'ella' como "[una fuente autorizada que procede tanto de la omnisciencia como de la imparcialidad (sabe todo, pero no está directamente involucrado)]” (Stern, 1991, p. 13). Por lo general, los portavoces son un vehículo para los argumentos informativos, puesto que son percibidos como personas veraces que aspiran a ganar la confianza de una audiencia cuando su objetivo es transmitir información sobre productos o usuarios en lugar de hablar de sí mismos (Stern, 1991). Por lo tanto, la forma mediante la que las distintas voces, en correlación con el pronombre personal tónico utilizado, se identifican frente a su audiencia y el pronombre utilizado en el mensaje para describir el producto llevan a plantear las siguientes preguntas:

PI 3: ¿Cuál es la evolución en el uso de los diferentes tipos de voces según la forma de identificación?

PI 4: ¿Cuál es la evolución en el uso de los diferentes tipos de voces según el pronombre del sujeto utilizado? 


\section{DISERTACIONES}

ESTUDIOS

\section{Metodología}

Para desarrollar este trabajo, se ha optado por una metodología de naturaleza cuantitativa de secuencia polietápica basada en la técnica del análisis de contenido que permite una descripción objetiva y sistemática del contenido manifiesto (Berelson, 1952) de cuñas publicitarias. En este caso, se trata de dos muestras referidas a los años 2009 y 2016.

Siguiendo un criterio estratégico intencional, el mes elegido para la obtención de las muestras iniciales fue junio, por ser este uno de los de mayor ocupación publicitaria de acuerdo con el estudio de inversión de referencia del sector Infoadex. Para la selección de las emisoras comerciales que emiten en español, de difusión nacional y de mayor audiencia, se tomaron los datos de la oleada del Estudio General de Medios (EGM) coincidentes con los primeros meses de los años analizados (Almc, 2009, 2016). En ambos casos, las emisoras fueron las mismas, excepto en dos. Radio generalista: Cadena SER, Onda Cero y Cadena COPE; Punto Radio solo aparece en la muestra de 2009. Radio temática: C40, Dial, Europa Fm, C100, Kiss Fm, Máxima Fm, Radio Olé y M80; Rock Fm solo aparece en la muestra de 2016.

\section{Procedimiento}

Se registraron las 24 horas de emisión de las 24 emisoras en cuatro semanas, de lunes a viernes en días consecutivos (martes, miércoles y jueves), en un dispositivo de almacenamiento externo de memoria. Mediante un programa informático de audio (Sony Sound Forge Audio Studio 9.0) se analizaron las 576 horas (288 horas/año) de la programación emitida para detectar las cuñas publicitarias y configurar el corpus inicial del análisis.

En primer lugar, se hizo la selección solo de aquellas emisoras cuyo ámbito de emisión fuera nacional, desechando la publicidad local. Aunque la radio es considerada un medio local por la cantidad de emisoras existentes en España con esta cobertura geográfica, entendimos que la investigación debía centrarse en las de difusión nacional, como suele hacerse en otros medios (prensa, televisión, revistas, etc.), para poder, posteriormente, realizar estudios comparativos con los mismos parámetros de difusión. En una segunda fase no se consideraron las cuñas de ofertas y promociones, ya que están basadas en el precio, buscan un incremento del consumo en un período de tiempo determinado -corto, por lo general-y su emisión tiene una limitación temporal muy concreta, y esto podría suponer un sesgo para los resultados. Se obtuvieron un total de 1664 cuñas publicitarias en el año 2009 y 3909 en 2016.

En una tercera fase se consideraron solo los anuncios en los que el producto estaba descrito por una voz que representa a un tercero o anunciante. Tras este filtrado, las muestras quedaron integradas por 1401 anuncios en 2009 y 2357 en 2016.

\section{Variables}

Los antecedentes registrados en estudios reseñados en la bibliografía y las preguntas de investigación han sido la base sobre la que se han operativizado las siguientes variables con sus correspondientes atributos:

a. Año. (1) 2009, (2) 2016. 


\section{DISERTACIONES}

ESTUDIOS

El papel del sonido en la comunicación: contribución, funciones y efectos

ISSN: 1856-9536

Doi: http://dx.doi.org/10.12804/revistas.urosario.edu.co/disertaciones/a.6786

Volumen 12, Número 2 / Julio-diciembre 2019

Versión PDF para imprimir desde

http://revistas.urosario.edu.co/index.php/disertaciones

b. Tipo de voz. La voz protagonista del mensaje que encarna al anunciante se ha clasificado según los siguientes atributos (Belch \& Belch, 2013; Fleck, et al., 2014; Friedman, et al., 1976; Stafford, et al., 2002): famoso de diferentes ámbitos de la (1) moda, (2) cine, (3) música, (4) deporte, de las (5) noticias y medios de comunicación, (6) empleado de la empresa, (7) experto profesional, (8) consumidor, (9) locutor.

c. Tipo de anunciante. (1) Multinacional, (2) Nacional.

d. Macrocategoría de producto. El contenido de esta variable se fundamenta en los criterios con los que Infoadex (estudio anual de inversión publicitaria) tipifica cualquier anuncio según áreas generales de mercado donde opera el objeto de consumo. Sin embargo, esa clasificación resulta poco operativa debido a su enorme dispersión, dificultando estudios cuantitativos donde las variables constan de muchos atributos. De tal forma, replicamos la clasificación realizada por Perelló Oliver y Muela Molina (2013), cuya propuesta consta de una nueva variable que integra de forma más operativa las tipologías de productos relacionadas con el discurso publicitario. Se trata de la variable 'macrocategoría de producto', en cuyo proceso de construcción se han aplicado dos criterios (Perelló Oliver, 2009): el primero pretende mejorar la heterogeneidad entre los distintos atributos que la integran; y el segundo, garantizar la homogeneidad interna de estos en términos de las categorías iniciales con las que trabaja Infoadex. De este modo, se ha construido una variable con cinco atributos que ha permitido caracterizar de una manera más operativa el contenido de la muestra. Con estos criterios de agrupación, la variable 'macrocategoría' de producto queda integrada por los cinco atributos siguientes: (1) hogar y vivienda, que comprende las categorías: alimentación, bebidas, construcción, distribución y restauración, energía, hogar, limpieza, telecomunicaciones e internet; (2) salud y belleza, resultado de integrar belleza e higiene, deportes y tiempo libre, objetos personales, salud, textil y vestimenta; (3) ocio y cultura agrupa: cultura, enseñanza y medios de comunicación, transporte, viajes y turismo; (4) finanzas: resultado de agregar finanzas y varios; (5) servicios, que integra: automoción; equipamiento de oficina, electrónica/informática y comercio; industrial, material de trabajo, agropecuario; servicios públicos y privados.

e. Identidad de la voz. La forma en como la voz se identifica para presentarse al oyente. Puede hacerlo de dos formas, a través del: (1) nombre y apellido, (2) cargo o desempeño profesional.

f. Pronombre del sujeto. (1) Yo, nosotros, cuando la fuente de información es el portavoz como un tercero que habla en representación del anunciante; (2) tú, vosotros, como los recursos lingüísticos para hacer referencia al destinatario; (3) él, ella, ellos es el recurso lingüístico cuando se refiere al anunciante.

Para preservar la confiabilidad de las muestras (Neuendorf, 2002), primero, un investigador ayudante realizó la escucha activa de las 576 horas de programación para configurar la muestra de cuñas publicitarias para cada año. Y, en una segunda fase, dos investigadores hicieron la codificación de las variables por separado. La única variable que generó algunas discrepancias fue la del 'pronombre del sujeto' por la presencia de varios pronombres utilizados en un anuncio. En estos casos, el problema se resolvió codificando según la persona del sujeto que aparecía con más frecuencia. Por último, para el filtrado de las muestras, la codificación de las unidades muestrales, el análisis de frecuencias y los tests de significatividad relativos a cada cruce de variables, se utilizó el programa estadístico spss versión 17. 


\section{DISERTACIONES}

ESTUDIOS

El papel del sonido en la comunicación: contribución, funciones y efectos ISSN: 1856-9536

Doi: http://dx.doi.org/10.12804/revistas.urosario.edu.co/disertaciones/a.6786 Volumen 12, Número 2/ Julio-diciembre 2019

Versión PDF para imprimir desde

http://revistas.urosario.edu.co/index.php/disertaciones

\section{Resultados}

Del análisis de los datos y en respuesta a la PI 1, se evidencia que la tipología de voces que protagonizan los anuncios publicitarios en radio presentan algunas particularidades relevantes desde la perspectiva del tipo de anunciante que los promueve (tabla 1) en el marco temporal que va desde 2009 a 2016. En primer lugar, la voz tipo 'locutor' adquiere un protagonismo creciente tanto en los anunciantes de perfil multinacional como en los nacionales. Las multinacionales incrementan el uso de este tipo de voz en un $42 \%$ en el período 2009-2016, mientras que los anunciantes nacionales lo hacen en un $56 \%$ en ese mismo período.

En segundo lugar, destaca el notable incremento de la voz protagonizada por el rol propio del 'empleado de la empresa', que en el caso de las multinacionales no tenía presencia en 2009, mientras que en 2016 se utilizó en 182 ocasiones (34\%). Los anunciantes nacionales multiplicaron por más de nueve veces el uso de este tipo de voz en el período 2009-2016.

La tercera voz con mayor protagonismo es la de los 'consumidores', cuya presencia se multiplicó por casi cuatro en el caso de las multinacionales, mientras que los anunciantes nacionales la redujeron en más de la mitad en el marco temporal de referencia, concretamente en un $54 \%$. En contraposición, las voces con menor protagonismo en la publicidad radiofónica corresponden a aquellas que son más relevantes en la mayor parte de la publicidad en otros medios, como son las propias de los actores cinematográficos, cantantes y deportistas de más actualidad y reconocimiento. Solo los personajes famosos propios de los medios de comunicación adquieren cierta presencia, pero, sobre todo, para los anunciantes nacionales.

Tabla 1. Evolución en el uso de la voz por tipo, tipología de anunciante y año

\begin{tabular}{|c|c|c|c|c|c|c|}
\hline \multirow{4}{*}{ Tipo de voz } & \multicolumn{4}{|c|}{ Tipo de anunciante } & \multirow{2}{*}{\multicolumn{2}{|c|}{ Total }} \\
\hline & \multicolumn{2}{|c|}{ Multinacional } & \multicolumn{2}{|c|}{ Nacional } & & \\
\hline & 2009 & 2016 & 2009 & 2016 & 2009 & 2016 \\
\hline & $\begin{array}{c}\text { Frecuencia } \\
\text { Horiz. \% }\end{array}$ & $\begin{array}{c}\text { Frecuencia } \\
\text { Horiz. \% }\end{array}$ & $\begin{array}{c}\text { Frecuencia } \\
\text { Horiz. \% }\end{array}$ & $\begin{array}{c}\text { Frecuencia } \\
\text { Horiz. \% }\end{array}$ & $\begin{array}{c}\text { Frecuencia } \\
\text { Horiz. \% }\end{array}$ & $\begin{array}{c}\text { Frecuencia } \\
\text { Horiz. \% }\end{array}$ \\
\hline \multirow{2}{*}{ Cine } & 0 & 0 & 0 & 8 & 0 & 8 \\
\hline &, 0 &, 0 &, 0 & 100,0 &, 0 & 100,0 \\
\hline \multirow{2}{*}{ Música } & 0 & 0 & 6 & 7 & 6 & 7 \\
\hline &, 0 &, 0 & 100,0 & 100,0 & 100,0 & 100,0 \\
\hline \multirow{2}{*}{ Deporte } & 12 & 6 & 10 & 0 & 22 & 6 \\
\hline & 54,45 & 100,0 & 45,5 &, 0 & 100,0 & 100,0 \\
\hline \multirow{2}{*}{ Moda } & 0 & 0 & 0 & 0 & 0 & 0 \\
\hline &, 0 &, 0 &, 0 &, 0 &, 0 &, 0 \\
\hline \multirow{2}{*}{ Noticias y medios } & 4 & 0 & 35 & 31 & 39 & 31 \\
\hline & 10,3 &, 0 & 89,7 & 100,0 & 100,0 & 100,0 \\
\hline \multirow{2}{*}{$\begin{array}{l}\text { Empleado de la } \\
\text { empresa }\end{array}$} & 0 & 182 & 38 & 354 & 38 & 536 \\
\hline & 0 & 34,0 & 100,0 & 66,0 & 100,0 & 100,0 \\
\hline
\end{tabular}




\section{DISERTACIONES}

ESTUDIOS

El papel del sonido en la comunicación: contribución, funciones y efectos ISSN: 1856-9536

Doi: http://dx.doi.org/10.12804/revistas.urosario.edu.co/disertaciones/a.6786 Volumen 12, Número 2/ Julio-diciembre 2019

Versión PDF para imprimir desde

http://revistas.urosario.edu.co/index.php/disertaciones

\begin{tabular}{|c|c|c|c|c|c|c|}
\hline \multirow{4}{*}{ Tipo de voz } & \multicolumn{4}{|c|}{ Tipo de anunciante } & \multirow{2}{*}{\multicolumn{2}{|c|}{ Total }} \\
\hline & \multicolumn{2}{|c|}{ Multinacional } & \multicolumn{2}{|c|}{ Nacional } & & \\
\hline & 2009 & 2016 & 2009 & 2016 & 2009 & 2016 \\
\hline & $\begin{array}{c}\text { Frecuencia } \\
\text { Horiz. \% }\end{array}$ & $\begin{array}{c}\text { Frecuencia } \\
\text { Horiz. \% }\end{array}$ & $\begin{array}{c}\text { Frecuencia } \\
\text { Horiz. \% }\end{array}$ & $\begin{array}{c}\text { Frecuencia } \\
\text { Horiz. \% }\end{array}$ & $\begin{array}{c}\text { Frecuencia } \\
\text { Horiz. \% }\end{array}$ & $\begin{array}{c}\text { Frecuencia } \\
\text { Horiz. \% }\end{array}$ \\
\hline \multirow{2}{*}{ Experto profesional } & 1 & 5 & 28 & 1 & 29 & 6 \\
\hline & 3,4 & 83,3 & 96,6 & 16,7 & 100,0 & 100,0 \\
\hline \multirow{2}{*}{ Consumidor } & 22 & 85 & 225 & 121 & 247 & 206 \\
\hline & 8,9 & 41,3 & 77,2 & 58,7 & 100,0 & 100,0 \\
\hline \multirow{2}{*}{ Locutor } & 278 & 395 & 742 & 1162 & 1020 & 1557 \\
\hline & 27,3 & 25,4 & 72,7 & 74,6 & 100,0 & 100,0 \\
\hline \multirow{2}{*}{ Total } & 317 & 673 & 1084 & 2184 & 1401 & 2357 \\
\hline & 22,6 & 23,6 & 77,4 & 76,4 & 100,0 & 100,0 \\
\hline
\end{tabular}

Nota: Tipo de voz \& tipo de anunciante \& 2009. Música: $\chi^{2}(1, N=1604)=1,566, p<0,221 \mid$ Deporte: $\chi^{2}(1, N=1604)=15,661$, $\mathrm{p}<0,001 \mid$ Noticias y medios: $\chi^{2}(1, \mathrm{~N}=1604)=2,629, \mathrm{p}<0,105 \mid$ Empleado de la empresa: $\chi^{2}(1, \mathrm{~N}=1604)=10,120, \mathrm{p}<0,001 \mid$ Experto profesional: $\chi^{2}(1, N=1604)=5,327, p<0,021 \mid$ Consumidor: $\chi^{2}(1, N=1604)=24,524, p<0,001 \mid$ Locutor: $\chi^{2}(1, N=1604)$ $=74,942, p<0,001$.

Tipo de voz \& tipo de anunciante \& 2016. Cine: $\chi^{2}(1, N=2376)=3,155, p<0,075 \mid$ Música: $\chi^{2}(1, N=2376)=2,769, p<0,096$ | Deporte: $\chi^{2}(1, N=2376)=15,253, p<0,001 \mid$ Noticias y medios: $\chi^{2}(1, N=2376)=12,387, p<0,001 \mid$ Empleado de la empresa: $\chi^{2}$ $(1, N=2376)=10,979, p<0,001 \mid$ Experto profesional: $\chi^{2}(1, N=2376)=8,987, p<0,003 \mid$ Consumidor: $\chi^{2}(1, N=2376)=18,733, p$ $<0,001$ | Locutor: $\chi^{2}(1, N=2376)=18,904, p<0,001$.

Desagregando por la variable macrocategoría de producto (tabla 2) para dar respuesta a la PI 2, las principales voces protagonistas de la publicidad radiofónica presentan comportamientos muy dispares, aunque se constata que la voz 'locutor' es la que más crece en la mayor parte de las categorías de producto referidas a lo largo del período 2009-2016. Esta voz multiplica por casi tres su presencia en los anuncios de 'salud y belleza', y en más de dos veces y media en los anuncios de 'servicios'. En las categorías de 'ocio y cultura' y 'finanzas', su protagonismo se incrementa en un $22 \%$ y un $44 \%$, respectivamente.

Esta tendencia al alza es la que también caracteriza a la voz que reproduce al 'empleado de la empresa', que crece muy notablemente en todos los productos y servicios. Tanto es así que pasa de acumular 38 casos en 2009 a 536 en 2016, multiplicando así 14 veces su presencia.

Sin embargo, en el caso de la voz 'consumidor', esta presenta una clara tendencia a la baja en todas las categorías de producto, a excepción de lo que ocurre en la categoría de 'servicios', donde se multiplica por más de tres a lo largo del período de referencia.

Tal y como se ha señalado anteriormente, la presencia de voces vinculadas al cine, la música, el deporte o la moda en relación con esta tipología de productos y servicios es muy marginal o simplemente nula. 


\section{DISERTACIONES}

ESTUDIOS

El papel del sonido en la comunicación: contribución, funciones y efectos ISSN: $1856-9536$

Doi: http://dx.doi.org/10.12804/revistas.urosario.edu.co/disertaciones/a.6786 Volumen 12, Número 2 / Julio-diciembre 2019

Versión PDF para imprimir desde

http://revistas.urosario.edu.co/index.php/disertaciones

Tabla 2. Evolución en el uso de la voz por tipo, macrocategoría de producto y año

\begin{tabular}{|c|c|c|c|c|c|c|c|c|c|c|c|c|}
\hline \multirow{4}{*}{ Tipo de voz } & \multicolumn{10}{|c|}{ Macrocategoría de producto } & \multirow{2}{*}{\multicolumn{2}{|c|}{ Total }} \\
\hline & \multicolumn{2}{|c|}{ Hogar y vivienda } & \multicolumn{2}{|c|}{ Salud y belleza } & \multicolumn{2}{|c|}{ Ocio y cultura } & \multicolumn{2}{|c|}{ Finanzas } & \multicolumn{2}{|c|}{ Servicios } & & \\
\hline & 09 & 16 & 09 & 16 & 09 & 16 & 09 & 16 & 09 & 16 & 09 & 16 \\
\hline & Fr. $\%$ * & Fr. \% & Fr. $\%$ & Fr. \% & Fr. \% & Fr. $\%$ & Fr. $\%$ & Fr. $\%$ & Fr. $\%$ & Fr. $\%$ & Fr. $\%$ & Fr. $\%$ \\
\hline \multirow{2}{*}{ Cine } & 0 & 8 & 0 & 0 & 0 & 0 & 0 & 0 & 0 & 0 & 0 & 8 \\
\hline & 0 & 100,0 &, 0 & 0 & 0 &, 0 &, 0 & 0 & 0 &, 0 & 0 & 100,0 \\
\hline \multirow{2}{*}{ Música } & 6 & 0 & 0 & 0 & 0 & 0 & 0 & 0 & 0 & 7 & 6 & 7 \\
\hline & 100,0 & 0 &, 0 &, 0 & 0 & 0 &, 0 &, 0 & 0 & 0 & 100,0 & 100,0 \\
\hline \multirow{2}{*}{ Deporte } & 0 & 0 & 10 & 0 & 0 & 0 & 12 & 5 & 0 & 1 & 22 & 6 \\
\hline & 0 &, 0 & 45,5 & 0 &, 0 & 0 & 54,5 & 83,3 & 0 & 16,7 & 100,0 & 100,0 \\
\hline \multirow{2}{*}{ Moda } & 0 & 0 & 0 & 0 & 0 & 0 & 0 & 0 & 0 & 0 & 0 & 0 \\
\hline & 0 & 0 &, 0 &, 0 & 0 & 0 & 0 &, 0 & 0 & 0 &, 0 &, 0 \\
\hline \multirow{2}{*}{$\begin{array}{l}\text { Noticias y } \\
\text { medios }\end{array}$} & 7 & 0 & 32 & 12 & 0 & 3 & 0 & 2 & 0 & 14 & 39 & 31 \\
\hline & 17,9 &, 0 & 82,1 & 38,7 & ,0 & 9,7 &, 0 & 6,5 &, 0 & 45,2 & 100,0 & 100,0 \\
\hline \multirow{2}{*}{$\begin{array}{l}\text { Empleado de } \\
\text { la empresa }\end{array}$} & 0 & 152 & 1 & 26 & 5 & 43 & 0 & 145 & 32 & 170 & 38 & 536 \\
\hline & , 0 & 28,4 & 2,6 & 4,9 & 13,2 & 8,0 &, 0 & 27,1 & 84,2 & 37,1 & 100,0 & 100,0 \\
\hline \multirow{2}{*}{$\begin{array}{l}\text { Experto } \\
\text { profesional }\end{array}$} & 0 & 0 & 25 & 6 & 3 & 0 & 1 & 0 & 0 & 0 & 29 & 6 \\
\hline & 0 & 0 & 86,2 & 100,0 & 10,3 & 0 & 3,4 & 0 & 0 & 0 & 100,0 & 100,0 \\
\hline \multirow{2}{*}{ Consumidor } & 42 & 25 & 73 & 49 & 41 & 29 & 60 & 0 & 31 & 103 & 247 & 206 \\
\hline & 17,0 & 12,1 & 29,6 & 23,8 & 16,6 & 14,1 & 24,3 &, 0 & 12,6 & 50,0 & 100,0 & 100,0 \\
\hline \multirow{2}{*}{ Locutor } & 264 & 213 & 97 & 273 & 257 & 315 & 238 & 342 & 164 & 414 & 1020 & 1557 \\
\hline & 25,9 & 13,7 & 9,5 & 17,5 & 25,2 & 50,2 & 23,8 & 22,0 & 16,1 & 26,6 & 100,0 & 100,0 \\
\hline \multirow{2}{*}{ Total } & 319 & 398 & 238 & 366 & 306 & 390 & 311 & 494 & 227 & 709 & 1401 & 2357 \\
\hline & 22,8 & 16,9 & 17,0 & 15,5 & 21,8 & 16,5 & 22,2 & 21,0 & 16,2 & 30,1 & 100,0 & 100,0 \\
\hline
\end{tabular}

* Frecuencia y porcentaje horizontal.

Nota: Tipo de voz \& macrocategoría de producto \& 2009. Música: $\chi^{2}(4, N=1604)=19,332, p<0,001 \mid$ Deporte: $\chi^{2}(4, N=1604)=$ 37,455, $\mathrm{p}<0,001 \mid$ Noticias y medios: $\chi^{2}(4, \mathrm{~N}=1604)=120,162, \mathrm{p}<0,001 \mid$ Empleado de la empresa: $\chi^{2}(4, \mathrm{~N}=1604)=110,244, \mathrm{p}$ $<0,001 \mid$ Experto profesional: $\chi^{2}(4, N=1604)=98,411, p<0,001 \mid$ Consumidor: $\chi^{2}(4, N=1604)=41,136, p<0,001 \mid$ Locutor: $\chi^{2}(4$, $\mathrm{N}=1604)=172,394, \mathrm{p}<0,001$.

Tipo de voz \& macrocategoría de producto \& 2016. Cine: $\chi^{2}(4, N=2376)=39,893, p<0,006 \mid$ Música: $\chi^{2}(4, N=2376)=16,574$, $\mathrm{p}<0,002$ | Deporte: $\chi^{2}(4, \mathrm{~N}=2376)=14,638, \mathrm{p}<0,001 \mid$ Noticias y medios: $\chi^{2}(4, \mathrm{~N}=2376)=21,436, \mathrm{p}<0,001 \mid$ Empleado de la empresa: $\chi^{2}(4, N=2376)=154,895, p<0,001 \mid$ Experto profesional: $\chi^{2}(4, N=2376)=30,915, p<0,001 \mid$ Consumidor: $\chi^{2}(4, N=$ $2376)=89,366, p<0,001 \mid$ Locutor: $\chi^{2}(4, N=2376)=88,040, p<0,001$

En la tabla 3, que responde a la PI 3, se desglosa la forma en la que las voces protagonistas del estudio configuran su propia identidad ante el oyente. Del conjunto de los casos estudiados, solo 241 en 2009 y 88 en 2016 concretan esa identidad bajo un nombre propio específico o mediante una filiación concreta a un puesto o cargo. Es importante destacar que, a pesar del notable aumento de la inversión publicitaria evidenciada por el incremento en el número de cuñas emitidas en dos momentos temporales distintos, esta fórmula de identificación de las voces ha perdido muchísimo peso. Todas las tipologías de voces ven diluido el recurso de identificación, a excepción del 'cine', que, a pesar de ello, podemos considerar un dato anecdótico. 
Con todo, es la voz que reproduce el rol del 'empleado de la empresa' la que con más asiduidad tiende a identificarse a través de un nombre propio y/o mediante el cargo de responsabilidad que ocupa en la organización. Y eso ocurre tanto en 2009 como en 2016.

Tabla 3. Evolución en el uso de la voz por tipo, identidad y año

\begin{tabular}{|c|c|c|c|c|c|c|}
\hline & \multicolumn{4}{|c|}{ Identidad } & \multirow{2}{*}{\multicolumn{2}{|c|}{ Total }} \\
\hline & \multicolumn{2}{|c|}{ Nombre } & \multicolumn{2}{|c|}{ Cargo } & & \\
\hline & 2009 & 2016 & 2009 & 2016 & 2009 & 2016 \\
\hline & $\mathrm{Fr}$. & Fr. & Fr. & $\mathrm{Fr}$. & $\mathrm{Fr}$. & Fr. \\
\hline & $\%^{*}$ & $\%$ & $\%$ & $\%$ & $\%$ & $\%$ \\
\hline \multirow{2}{*}{ Cine } & 0 & 8 & 0 & 0 & 0 & 8 \\
\hline & 0 & 100,0 & 0 & 0 & 0 & 100,0 \\
\hline \multirow{2}{*}{ Música } & 6 & 7 & 6 & 0 & 12 & 7 \\
\hline & 50 & 100,0 & 50,0 & , 0 & 100,0 & 100,0 \\
\hline \multirow[b]{2}{*}{ Deporte } & 22 & 6 & 22 & 0 & 44 & 6 \\
\hline & 50,0 & 100,0 & 50,0 & 0 & 100,0 & 100,0 \\
\hline \multirow{2}{*}{ Moda } & 0 & 0 & 0 & 0 & 0 & 0 \\
\hline &, 0 &, 0 &, 0 & 0 &, 0 &, 0 \\
\hline \multirow{2}{*}{ Noticias y medios } & 39 & 15 & 4 & 0 & 43 & 15 \\
\hline & 90,7 & 100,0 & 9,3 & 0 & 100,0 & 100,0 \\
\hline \multirow{2}{*}{$\begin{array}{l}\text { Empleado de la } \\
\text { empresa }\end{array}$} & 38 & 24 & 38 & 10 & 76 & 34 \\
\hline & 0 & 0 & 0 & 0 &, 0 & 0 \\
\hline \multirow{2}{*}{ Experto profesional } & 25 & 5 & 28 & 6 & 53 & 11 \\
\hline & 47,17 & 45,45 & 52,83 & 54,55 & 100,0 & 100,0 \\
\hline \multirow{2}{*}{ Consumidor } & 13 & 5 & 0 & 2 & 13 & 7 \\
\hline & 100,0 & 71,4 & 0 & 28,6 & 100,0 & 100,0 \\
\hline \multirow{2}{*}{ Locutor } & 0 & 0 & 0 & 0 & 0 & 0 \\
\hline &, 0 &, 0 & 0 &, 0 &, 0 &, 0 \\
\hline \multirow{2}{*}{ Total } & 143 & 70 & 98 & 18 & 241 & 88 \\
\hline & 59,34 & 79,55 & 40,66 & 20,45 & 100,0 & 100,0 \\
\hline
\end{tabular}

${ }^{*}$ Frecuencia y porcentaje horizontal.

Nota: Tipo de voz \& nombre \& 2009. Música: $\chi^{2}(1, N=1604)=61,531, p<0,001 \mid$ Deporte: $\chi^{2}(1, N=1604)=227,895, p<0,001 \mid$ Noticias y medios: $\chi^{2}(1, N=1604)=408,384, p<0,001 \mid$ Empleado de la empresa: $\chi^{2}(1, N=1604)=397,659, p<0,001 \mid$ Experto profesional: $\chi^{2}(1, N=1604)=217,275, p<0,001 \mid$ Consumidor: $\chi^{2}(1, N=1604)=4,795, p<0,029$.

Tipo de voz \& nombre \& 2016. Cine: $\chi^{2}(1, N=2376)=186,946, p<0,001 \mid$ Música: $\chi^{2}(4, N=2376)=16,574, p<0,002 \mid$ Deporte: $\chi^{2}$ $(1, N=2376)=160,172, p<0,001 \mid$ Noticias y medios: $\chi^{2}(1, N=2376)=180,448, p<0,001 \mid$ Empleado de la empresa: $\chi^{2}(1, N=$ $2376)=1,461, p<0,227 \mid$ Experto profesional: $\chi^{2}(1, N=2376)=109,566, p<0,001 \mid$ Consumidor: $\chi^{2}(1, N=2376)=6,351, p<0,012$.

Tipo de voz \& cargo \& 2009. Música: $\chi^{2}(1, N=1604)=92,550, p<0,001 \mid$ Deporte: $\chi^{2}(1, N=1604)=342,783, p<0,001 \mid$ Noticias y medios: $\chi^{2}(1, N=1604)=1,198, p<0,274$ | Empleado de la empresa: $\chi^{2}(1, N=1604)=598,129, p<0,001$ | Experto profesional: $\chi^{2}(1, N=1604)=421,133, p<0,001$.

Tipo de voz \& cargo \& 2016. Empleado de la empresa: $\chi^{2}(1, N=2376)=0,927, p<0,336 \mid$ Experto profesional: $\chi^{2}(1, N=2376)=$ $414,340, p<0,001 \mid$ Consumidor: $\chi^{2}(1, N=2376)=0,339, p<0,561$. 


\section{DISERTACIONES}

ESTUDIOS

El papel del sonido en la comunicación: contribución, funciones y efectos ISSN: 1856-9536

Doi: http://dx.doi.org/10.12804/revistas.urosario.edu.co/disertaciones/a.6786 Volumen 12, Número 2/ Julio-diciembre 2019

Versión PDF para imprimir desde

http://revistas.urosario.edu.co/index.php/disertaciones

Tal y como se ha analizado en el marco teórico, la forma que toma el pronombre utilizado en el mensaje es un elemento que indica el grado de relación que establece el portavoz con el propio producto o servicio anunciado. La tabla 4 desglosa esa variable en relación con los distintos tipos de voces a lo largo del período estudiado a la vez que da respuesta a la PI 4. Se constata que el tipo de voz predominante, la del 'locutor', se decanta principalmente por el uso de la tercera persona del singular y del plural, tanto en el año 2009 (483, 42,9\% del total) como en 2016 (763, 49\% del total), al objeto de dar la mayor sensación de imparcialidad posible ante el oyente subrayando, así, su naturaleza basada en el anonimato.

Tabla 4. Evolución en el uso de la voz por tipo, pronombre del sujeto y año

\begin{tabular}{|c|c|c|c|c|c|c|c|c|}
\hline \multirow{4}{*}{ Tipo de voz } & \multicolumn{6}{|c|}{ Pronombre del sujeto } & \multirow{2}{*}{\multicolumn{2}{|c|}{ Total }} \\
\hline & \multicolumn{2}{|c|}{ Yo, nosotros } & \multicolumn{2}{|c|}{ Tú, vosotros } & \multicolumn{2}{|c|}{ Él, ella, ellos } & & \\
\hline & 2009 & 2016 & 2009 & 2016 & 2009 & 2016 & 2009 & 2016 \\
\hline & $\begin{array}{l}\text { Fr. } \\
\%^{*}\end{array}$ & $\begin{array}{l}\text { Fr. } \\
\%\end{array}$ & $\begin{array}{l}\text { Fr. } \\
\%\end{array}$ & $\begin{array}{l}\text { Fr. } \\
\%\end{array}$ & $\begin{array}{l}\text { Fr. } \\
\%\end{array}$ & $\begin{array}{l}\text { Fr. } \\
\%\end{array}$ & $\begin{array}{l}\text { Fr. } \\
\%\end{array}$ & $\begin{array}{l}\text { Fr. } \\
\%\end{array}$ \\
\hline \multirow{2}{*}{ Cine } & 0 & 0 & 0 & 8 & 0 & 0 & 0 & 8 \\
\hline &, 0 & , 0 & 0 & 100,0 & 0 &, 0 &, 0 & 100,0 \\
\hline \multirow{2}{*}{ Música } & 6 & 1 & 0 & 6 & 0 & 0 & 6 & 7 \\
\hline & 100,0 & 14,3 & 0 & 85,7 & 0 & 0 & 100,0 & 100,0 \\
\hline \multirow{2}{*}{ Deporte } & 22 & 6 & 0 & 0 & 0 & 0 & 22 & 6 \\
\hline & 100,0 & 100,0 &, 0 &, 0 &, 0 &, 0 & 100,0 & 100,0 \\
\hline \multirow{2}{*}{ Moda } & 0 & 0 & 0 & 0 & 0 & 0 & 0 & 0 \\
\hline & 0 & 0 & 0 & 0 & 0 & 0 & 0 & 0 \\
\hline \multirow{2}{*}{$\begin{array}{l}\text { Noticias y } \\
\text { medios }\end{array}$} & 35 & 4 & 0 & 4 & 4 & 23 & 39 & 31 \\
\hline & 89,7 & 12,9 &, 0 & 12,9 & 10,3 & 74,2 & 100,0 & 100,0 \\
\hline \multirow{2}{*}{$\begin{array}{l}\text { Empleado de } \\
\text { la empresa }\end{array}$} & 32 & 391 & 5 & 97 & 1 & 48 & 38 & 536 \\
\hline & 84,2 & 72,9 & 13,2 & 18,1 & 2,6 & 9,0 & 100,0 & 100,0 \\
\hline \multirow{2}{*}{$\begin{array}{l}\text { Experto } \\
\text { profesional }\end{array}$} & 4 & 1 & 14 & 0 & 11 & 5 & 29 & 6 \\
\hline & 13,8 & 16,7 & 18,3 &, 0 & 37,9 & 83,3 & 100 & 100,0 \\
\hline \multirow{2}{*}{ Consumidor } & 184 & 71 & 17 & 61 & 46 & 74 & 247 & 206 \\
\hline & 74,5 & 34,5 & 6,9 & 29,6 & 18,6 & 35,9 & 100,0 & 100,0 \\
\hline \multirow{2}{*}{ Locutor } & 271 & 52 & 311 & 742 & 438 & 763 & 1020 & 1557 \\
\hline & 26,6 & 3,3 & 30,5 & 47,7 & 42,9 & 49,0 & 100,0 & 100,0 \\
\hline \multirow{2}{*}{ Total } & 554 & 526 & 347 & 918 & 500 & 913 & 1401 & 2357 \\
\hline & 39,5 & 22,3 & 24,8 & 38,9 & 35,7 & 38,7 & 100,0 & 100,0 \\
\hline
\end{tabular}

* Frecuencia y porcentaje horizontal.

Nota: Tipo de voz \& pronombre del sujeto \& 2009. Música: $\chi^{2}(2, N=1064)=10,268, p<0,006 \mid$ Deporte: $\chi^{2}(2, N=1064)=38,029$, $\mathrm{p}<0,001 \mid$ Noticias y medios: $\chi^{2}(2, N=1064)=48,340, p<0,001 \mid$ Empleado de la empresa: $\chi^{2}(2, N=1064)=38,145, p<0,001 \mid$ Experto profesional: $\chi^{2}(2, N=1064)=8,240, p<0,016 \mid$ Consumidor: $\chi^{2}(2, N=1064)=180,756, p<0,001 \mid$ Locutor: $\chi^{2}(2, N=1064)$ $=148,530, p<0,001$.

Tipo de voz \& pronombre del sujeto \& 2016. Cine: $\chi^{2}(2, N=2376)=12,659, p<0,002 \mid$ Música: $\chi^{2}(2, N=2376)=6,900, p<0,032 \mid$ Deporte: $\chi^{2}(2, N=2376)=20,354, p<0,001 \mid$ Noticias y medios: $\chi^{2}(2, N=2376)=17,276, p<0,001 \mid$ Empleado de la empresa: $\chi^{2}$ $(2, N=2376)=995,335, p<0,001 \mid$ Experto profesional: $\chi^{2}(2, N=2376)=5,600, p<0,061 \mid$ Consumidor: $\chi^{2}(2, N=2376)=5318,699$, $\mathrm{p}<0,001$ | Locutor: $\chi^{2}(2, \mathrm{~N}=2376)=974,701, \mathrm{p}<0,001$. 


\section{DISERTACIONES}

ESTUDIOS

El papel del sonido en la comunicación: contribución, funciones y efectos

ISSN: $1856-9536$

Doi: http://dx.doi.org/10.12804/revistas.urosario.edu.co/disertaciones/a.6786

Volumen 12, Número 2 / Julio-diciembre 2019

Versión PDF para imprimir desde

http://revistas.urosario.edu.co/index.php/disertaciones

En el caso del tipo 'empleado de la empresa', es la primera persona la que adquiere el máximo protagonismo a la hora de vehicular el mensaje, tanto en 2009 como en 2016. Sin duda, el objetivo no es otro que transmitir una mayor implicación personal en el anuncio para ganar la máxima credibilidad ante la audiencia, garantizando verbalmente la calidad del producto o servicio que él mismo promociona. De hecho, el 72,9\% de las voces de este tipo en 2016 se articulaban en torno a la primera persona del singular/plural, mientras que en 2009 ese porcentaje ascendía al 84,2\%.

La voz 'consumidor', sin embargo, opta principalmente por el uso de la primera persona (184 veces en 2009, un $74 \%$ del total). Destaca que este recurso pierde mucho peso en el año 2016, y lo hace en favor del mayor uso de la tercera y la segunda persona.

\section{Conclusiones y discusión}

La voz es la principal protagonista de la publicidad radiofónica y, a pesar de ello, ha sido objeto de muy pocos estudios específicos a partir de muestras estadísticamente representativas. En este trabajo, sustentado precisamente en dos muestras que cumplen con los criterios pertinentes de fiabilidad muestral, se ha puesto de manifiesto un incremento notable de la presencia de los diferentes tipos de voces de referencia en el período temporal estudiado, que va desde 2009 a 2016. Si en el primero de los años, el total acumulado ascendía a 1401 voces, en 2016 esa cifra alcanzó los 2357 casos. Con todo, es importante detallar que han sido las cuñas protagonizadas por la voz de los empleados de las empresas las que más han crecido en términos relativos, ya que han pasado de acumular 38 casos en 2009 a un total de 536 en 2016, lo que implica que su presencia se ha multiplicado por 14. La voz de los consumidores es la que más peso relativo tiene en el conjunto de los dos años estudiados, acumulando 1020 casos en 2009 y 1557 en 2016, lo que supone un crecimiento del $52 \%$. El resto de los tipos de voces acumula una presencia notablemente menor en el conjunto de ambas muestras, destacando la marginalidad e irrelevancia de aquellas que en otros medios asumen un mayor protagonismo, como son las voces procedentes de personajes relacionados con el mundo del cine, la música, el deporte o la moda. En un punto bajo, pero necesariamente destacable, aparecen las voces relacionadas con los profesionales de los medios de comunicación del ámbito nacional, que acumulan apenas 39 casos en 2009 y 31 en 2016.

En cualquier caso, los resultados del trabajo refuerzan la hipótesis de partida en cuanto la publicidad en la radio muestra una clara involución como ya lo han demostrado trabajos previos (Muela Molina, 2001; Rodero Antón, 2008). Los protagonistas del mensaje remiten a personajes reales o suelen ser reproducciones fieles de la realidad (Muela Molina, 2007). El retroceso es incluso mayor cuando se toman como referencia las voces de los actores que representan personajes de ficción, que son aquellas excluidas de este trabajo. Así, frente a investigaciones previas en las que la ficción representaba casi un 30 \% (28,25\%) de las cuñas analizadas (Arcos Foix \& Perona Páez, 2011), en nuestro caso, se ha pasado de un $12,7 \%$ en 2009 a un 7,8\% en 2016, resultados a los que hay que sumar también las cuñas dramatizadas, es decir que, aunque no recurren a historias fantásticas, sí son dramatizaciones 0 recreaciones donde los personajes que describen el producto son otros diferentes a los que representan las voces analizadas en este trabajo. Ello da cuenta de que la publicidad radiofónica sigue desaprovechando cada vez con más evidencia el potencial creativo e imaginativo del medio (Muela Molina, 2007). 


\section{DISERTACIONES}

ESTUDIOS

El papel del sonido en la comunicación: contribución, funciones y efectos

ISSN: 1856-9536

Doi: http://dx.doi.org/10.12804/revistas.urosario.edu.co/disertaciones/a.6786

Volumen 12, Número 2 / Julio-diciembre 2019

Versión PDF para imprimir desde

http://revistas.urosario.edu.co/index.php/disertaciones

También, en consonancia con trabajos previos, se ha constatado un predominio notable de la voz del locutor anónimo o straight announcer (Altstiel \& Grow, 2006) como voz institucional, "un empleo muy limitado de las posibilidades que ofrece la voz en la radio" (Rodero Antón, 2008, p. 13). Así mismo, las escasas dramatizaciones de supuestos gerentes de empresas, de expertos profesionales o de amistosos consumidores restan credibilidad a un mensaje que suena a artificioso y no auténtico. Esta apatía en el buen hacer de la publicidad radiofónica solo puede ser justificable si, como sostiene Cebrián Herreros (1998), el anunciante pretende que la autenticidad y fiabilidad que la audiencia percibe de la radio como medio informativo (Toharia, 2017) se proyecten en su producto o servicio.

El hecho de que en la publicidad de productos relacionados con la salud y la belleza, que suelen ser de alta implicación para el consumidor, predominen las voces de profesionales de los medios de comunicación a los que se les asocia un alto grado de credibilidad, demuestra una clara intencionalidad por parte del anunciante a la hora de seleccionar determinados personajes conocidos y de prestigio para incrementar el poder persuasivo de la publicidad a través de la ruta periférica (Petty \& Cacioppo, 1986; Petty, Cacioppo \& Schumann, 1983). Ello redunda en el hecho de que la radio ha sido tradicionalmente el medio o el refugio para la publicidad de los productos milagro. También cabe destacar que los profesionales de los medios prescriban el producto en primera persona del singular, lo que implica un mayor acercamiento con el referente de la comunicación. Un aspecto que subraya una práctica poco ética, ya que el código del periodista no permite la colaboración de estos profesionales en la publicidad (Perelló Oliver \& Muela Molina, 2017). Y, por otro lado, enfatiza la intención del anunciante de utilizar un personaje famoso y de gran reconocimiento y prestigio social, como pueden ser periodistas o presentadores de televisión, para aprovechar su credibilidad informativa y mediática en beneficio de su producto. En el caso de la radio, por lo tanto, la credibilidad de la fuente a partir de las variables de confianza y experiencia (Ohanian, 1990) prima sobre la transferencia de los valores (McCracken, 1989) que puedan proyectar los personajes famosos en el producto dada la escasa presencia de estos últimos adscritos al ámbito de lo social más que al de los medios de comunicación.

Y también es destacable, por lo sorprendente del resultado, que los expertos profesionales recomienden un producto haciendo referencia al fabricante y lo hagan, por lo general, en segunda y en tercera persona, apelando al consumidor y al fabricante, respectivamente, alejándose del referente y sin apenas involucrarse en la recomendación. Cuando lo fácil y lo que deduce la lógica es que el consumidor recomiende el producto que consume desde su propia experiencia en primera persona, al igual que el experto profesional que avala la marca desde su conocimiento profesional.

Otro de los resultados sorprendentes del trabajo tiene como base la variable denominada identificación de la voz. En un medio carente de imagen como la radio, el uso de personajes que desempeñan un rol social o profesional requiere una rápida presentación a su audiencia para que el receptor pueda centrarse en el contenido del mensaje y no entretenerse en adivinar quién está promocionando un producto o servicio. Abunda en esa necesidad el hecho de que la radio es un medio distractor cuya escucha se compagina con otra actividad, con lo cual no solo es necesario que la voz se identifique rápidamente, sino que se repita más de una vez por si el receptor no prestó atención cuando debía. En este sentido, las voces de las personas más conocidas por la sociedad como los famosos pertenecientes al ámbito del cine y la música o los profesionales de los medios de comunicación, especialmente los que trabajan en radio y televisión, se suelen identificar solo a través del nombre, mientras que los expertos profesionales y los empleados o personas asociadas a la empresa fabricante se identifican, además de por el nombre, por el oficio que practican o el cargo que desempeñan en la organización. En cualquier caso, solo en el 15\% de los 


\section{DISERTACIONES}

ESTUDIOS

El papel del sonido en la comunicación: contribución, funciones y efectos

ISSN: 1856-9536

Doi: http://dx.doi.org/10.12804/revistas.urosario.edu.co/disertaciones/a.6786

Volumen 12, Número 2 / Julio-diciembre 2019

Versión PDF para imprimir desde

http://revistas.urosario.edu.co/index.php/disertaciones

casos en que un personaje describe el producto es identificado mediante su nombre y apellidos y/o a través de su profesión, porcentaje que ha disminuido en siete años a un 3,7\%. Esta variable que aporta originalidad al trabajo profundiza en la situación con la que agencias y creativos trabajan, y en el poco interés que pone un anunciante en la exigencia de una correcta colaboración de la persona que está avalando su producto o servicio.

En definitiva, el medio radio debe acometer un debate profundo respecto a la forma de hacer publicidad a partir de una contradicción que es necesario resolver. Por un lado, se constata una clara infrautilización de su potencial creativo y comercial, teniendo en cuenta la enorme consideración social y credibilidad que la audiencia profesa a la radio. Y, por otro, este trabajo ha puesto de manifiesto que la radio es el medio en el que son más habituales las prácticas creativas cuya finalidad es, en muchos casos, desarrollar campañas publicitarias irrespetuosas con el oyente, incluso en productos con alta implicación para los consumidores. La necesidad de encontrar un 'equilibrio de oro' entre la credibilidad del medio y la persuasión publicitaria será, sin duda, uno de los ejes futuros sobre los que pivotarán los trabajos que versen sobre este objeto de estudio. Es importante pasar de los diagnósticos al desarrollo de metodologías de producción creativa que exploten al máximo el potencial del medio y, a la vez, sean respetuosas con los derechos de los consumidores, como muchos investigadores llevan reclamando décadas. Además, son imprescindibles las aproximaciones experimentales a la percepción que los consumidores tienen de los usos de la voz en las comunicaciones comerciales radiofónicas.

Es importante señalar en este punto las limitaciones en las que incurre un trabajo que pivota sobre dos muestras que, aun siendo estadísticamente representativas y garantistas desde el punto de vista del rigor con el que se han configurado, presentan algunos sesgos inevitables. El primero de ellos tiene que ver con el tamaño de las muestras utilizadas. Si bien es cierto que ambas son perfectamente homogéneas en relación con los criterios aplicados en su composición, el hecho de que se hayan construido a partir de una decisión estratégico intencional referida a la selección de los días concretos del año en los que registrar las muestras, y además en dos momentos temporales distintos, implica distintos volúmenes de unidades muestrales que pueden generar sesgos. Especialmente si tenemos en cuenta que 2009 fue un año de una notable reducción de la inversión publicitaria por el efecto de la crisis, mientras que 2016 presentó claros síntomas de recuperación en esa variable. Esto ha determinado volúmenes dispares de muestra a pesar de que se han aplicado los mismos criterios de elaboración. En el futuro convendrá introducir una fase más en la configuración polietápica de la muestra para que culmine con una equiparación del volumen de esta, incorporando la idea de que un mayor volumen no necesariamente garantiza mejor la representatividad estadística.

La segunda limitación deriva de la anterior en el sentido de que, dado el enorme volumen de ambas muestras, especialmente la referida al año 2016, hay un porcentaje de casos relativamente importante, en dicho año en concreto, que son de muy difícil encaje en el proceso de codificación de las variables diseñadas para el trabajo. Este será otro elemento que podrá resolverse con el uso de muestras más manejables.

Este trabajo avanza en el análisis del uso ineficaz y del no aprovechamiento del potencial comunicativo y creativo del medio radio como soporte publicitario, centrando el objeto de estudio en el tipo de voz, su identificación e implicación con el producto. Trabajos futuros que ya se han sugerido párrafos antes deben complementarse con otros estudios comparativos en distintos medios de comunicación y en otros países que permitan comprobar si el inmovilismo que padece la publicidad en la radio es endémico y característico del medio sonoro o también sucede en otros medios y en distintas áreas geográficas. 


\section{DISERTACIONES}

ESTUDIOS

\section{Referencias}

1. Altstiel, T., \& Grow, J. (2006). Advertising strategy. Creative tactics from the outside/in. Thousand Oaks, cA: SAGE Publications.

2. Amos, C., Holmes, G., \& Strutton, D. (2008). Exploring the relationship between celebrity endorser effects and advertising effectiveness. International Journal of Advertising, 27(2), 209-234.

3. Arcos Foix, N. A., \& Perona Páez, J. J. (2011). Modalidades, usos y presencia de la ficción como recurso creativo en la publicidad radiofónica. Anàlisi: quaderns de comunicació i cultura, (43), 1-19.

4. Asociación para la Investigación de Medios de Comunicación (Almc). (2009). Resumen general del Estudio General de Medios (EGM) abril 2008 a marzo 2009. Recuperado de http://www.aimc.es/spip.php?action=acceder_document\&arg=100\&cle=113d35dc5dc25f66cfe14b414f82816f2fc2ce1f\&file=pdf\%2Fresumegm109.pdf

5. Asociación para la Investigación de Medios de Comunicación (AIMc). (2016). Resumen general del Estudio General de Medios (EGM) abril 2015 a marzo 2016. Recuperado de http://www.aimc.es/spip.php?action=acceder_ document\&arg=3077\&cle=28996732bec589ddba1b4818789266af2af5377d\&file=pdf\%2Fresumegm116.pdf

6. Belch, G. E., \& Belch, M. A. (2013). A content analysis study of the use of celebrity endorsers in magazine advertising. International Journal of Advertising, 32(3), 369-389.

7. Berelson, B. (1952). Content analysis in communication research. Nueva York: Haffner Press.

8. Biswas, D., Biswas, A., \& Das, N. (2006). The differential effects of celebrity and expert endorsements on consumer risk perceptions. The role of consumer knowledge, perceived congruency, and product technology orientation. Journal of Advertising, 35(2), 17-31.

9. Black, B., \& Choi, J. (2013). An analysis of celebrity endorsements in magazine advertisements. Journalism and Mass Communication, 3(10), 615-624.

10. Buchholz, L. M., \& Smith, R. E. (1991). The role of consumer involvement in determining cognitive response to broadcast advertising. Journal of Advertising, 20(1), 4-17.

11. Cebrián Herreros, M. (1998). Información audiovisual: concepto, técnica, expresión y aplicaciones. Madrid: Síntesis.

12. Choi, S. M., Lee, W. N., \& Kim, H. J. (2005). Lessons from the rich and famous: a cross-cultural comparison of celebrity endorsement in advertising. Journal of Advertising, 34(2), 85-98.

13. Doss, S. (2011). The transference of brand attitude: the effect on the celebrity endorser. Journal of Management and Marketing Research, 7(1), 58-70.

14. Eisend, M., \& Langner, T. (2010). Immediate and delayed advertising effects of celebrity endorsers' attractiveness and expertise. International Journal of Advertising, 29(4), 527-546.

15. Fleck, N., Korchia, M., \& Le Roy, I. (2012). Celebrities in advertising: looking for congruence or likability? Psychology \& Marketing, 29(9), 651-662.

16. Fleck, N., Michel, G., \& Zeitoun, V. (2014). Brand personification through the use of spokespeople: an exploratory study of ordinary employees, cEOS, and celebrities featured in advertising. Psychology \& Marketing, 31(1), 84-92. 


\section{DISERTACIONES}

ESTUDIOS

El papel del sonido en la comunicación: contribución, funciones y efectos

ISSN: $1856-9536$

Doi: http://dx.doi.org/10.12804/revistas.urosario.edu.co/disertaciones/a.6786

Volumen 12, Número 2 / Julio-diciembre 2019

Versión PDF para imprimir desde

http://revistas.urosario.edu.co/index.php/disertaciones

17. Friedman, H. H., Termini, S., \& Washington, R. (1976). The effectiveness of advertisements utilizing four types of endorsers. Journal of Advertising, 5(3), 22-24.

18. Gaied, A. M., \& Rached, K. S. B. (2010). The persuasive effectiveness of famous and non famous endorsers in advertising. IBIMA Business Review, 2010, 1-14.

19. Hsu, C. K., \& McDonald, D. (2002). An examination on multiple celebrity endorsers in advertising. Journal of Product \& Brand Management, 11(1), 19-29.

20. Kertz, C. L., \& Ohanian, R. (1992). Source credibility, legal liability, and the law of endorsements. Journal of Public Policy \& Marketing, 11(1), 12-23.

21. Khatri, P. (2006). Celebrity endorsement: a strategic promotion perspective. Indian Media Studies Journal, 1(1), 25-37.

22. Knapp, M. L. (1980). Essentials of nonverbal communication. Nueva York: Holt, Rinehart and Winston.

23. McCracken, G. (1989). Who is the celebrity endorser? Cultural foundations of the endorsement process. Journal of Consumer Research, 16(3), 310-321.

24. Mittelstaedt, J. D., Riesz, P. C., \& Burns, W. J. (2000). Why are endorsements effective? Sorting among theories of product and endorser effects. Journal of Current Issues \& Research in Advertising, 22(1), 55-65.

25. Muela Molina, C. (2001). La publicidad radiofónica en España. Análisis creativo de sus mensajes. Madrid: ElunsA.

26. Muela Molina, C. (2007). El surrealismo en la cuña radiofónica. La dimensión de lo absurdo al servicio de la comunicación comercial. Espéculo: Revista de Estudios Literarios, (37), 83.

27. Muela Molina, C. (2010). La representación de los géneros informativos en la publicidad radiofónica. Sphera Pública, 10, 167-178.

28. Neuendorf, K. A. (2002). The content analysis guidebook (Vol. 300). Thousand Oaks, CA: SAGE Publications.

29. Ohanian, R. (1990). Construction and validation of a scale to measure celebrity endorsers' perceived expertise, trustworthiness, and attractiveness. Journal of Advertising, 19(3), 39-52.

30. Perelló Oliver, S., \& Muela Molina, C. (2013). Análisis de contenido de la publicidad radiofónica en España. methaodos. revista de ciencias sociales, 1(1), 33-52.

31. Perelló Oliver, S. (2009). Metodología de la investigación social. Madrid: Dykinson.

32. Perelló Oliver, S., \& Muela Molina, C. (2017). Radio mentions: an analysis of radio personalities and ethical behavior. International Journal of Communication, 11, 3566-3586.

33. Perona Páez, J. J. (2007). Formatos y estilos publicitarios en el prime-time radiofónico español: infrautilización y sequía de ideas. zer: Revista de Estudios de Comunicación, 12(23), 219-242.

34. Perona Páez, J. J., \& Barbeito Veloso, M. (2008). El lenguaje radiofónico en la publicidad del prime time generalista. Los anuncios en la "radio de las estrellas". Telos: Cuadernos de Comunicación, Tecnología y Sociedad, (77), 115-124.

35. Petty, R. E., \& Cacioppo, J. T. (1986). Communication and persuasion. Central and peripheral routes to attitude change. Nueva York: Springer-Verlag.

36. Petty, R. E., Cacioppo, J. T., \& Schumann, D. (1983). Central and peripheral routes to advertising effectiveness: the moderating role of involvement. Journal of Consumer Research, 10(2), 135-146.

37. Piñeiro Otero, T. (2011). Representaciones de género en la publicidad radiofónica. Un análisis comparativo. Vivat Academia, 115, 71-94. 


\section{DISERTACIONES}

38. Priester, J. R., \& Petty, R. E. (2003). The influence of spokesperson trustworthiness on message elaboration, attitude strength, and advertising effectiveness. Journal of Consumer Psychology, 13(4), 408-421.

39. Rodero Antón, E. (2008). Publicidad en radio: publicidad, sí, pero no radiofónica. Área Abierta, 20, 1-16.

40. Rodero, E., Larrea, O., \& Vázquez, M. (2010). Voces masculinas y femeninas en la locución de cuñas publicitarias. Estudio sobre la efectividad y su adecuación al producto. En El poder creativo de la palabra. Actas del I Congreso Publirradio (pp. 281-294). Madrid: Actas Icono (Vol. 14). Recuperado de http://documents.emmarodero. com/022-vocesmasculinasyfemeninas.pdf

41. Silvera, D. H., \& Austad, B. (2004). Factors predicting the effectiveness of celebrity endorsement advertisements. European Journal of Marketing, 38(11/12), 1509-1526.

42. Stafford, M. R., Stafford, T. F., \& Day, E. (2002). A contingency approach: the effects of spokesperson type and service type on service advertising perceptions. Journal of Advertising, 31(2), 17-34.

43. Stephens, N., \& Faranda, W. T. (1993). Using employees as advertising spokespersons. Journal of Services Marketing, 7(2), 36-46.

44. Stern, B. B. (1991). Who talks advertising? Literary theory and narrative "point of view". Journal of Advertising, 20(3), 9-22.

45. Stout, P. A., \& Moon, Y. S. (1990). Use of endorsers in magazine advertisements. Journalism \& Mass Communication Quarterly, 67(3), 536-546.

46. Toharia, J. J. (Coord.). (2017). Pulso de España 2016. Un informe sociológico. Recuperado de http://metroscopia.org/confianza-en-las-instituciones-espana-en-perspectiva-comparada/

47. Tulloch, J. (2014). Ethics, trust and the first person in the narration of long-form journalism. Journalism, 15(5), 629-638. 